\title{
A Mathematical Study to Gout Symptoms
}

\author{
Shuixian Yan 1,2, Victor Moreno3 ${ }^{3}$ Sanling Yuan ${ }^{4}$, Baojun Song 5 \\ ${ }^{1}$ Business School, University of Shanghai for Science and Technology, Shanghai, China \\ ${ }^{2}$ Key Laboratory of Jiangxi Province for Numerical Simulation and Emulation Techniques, Gannan Normal University, Ganzhou, \\ China \\ ${ }^{3}$ Simon A. Levin Mathematical, Computational and Modeling Sciences Center, Arizona State University, Tempe, AZ, USA \\ ${ }^{4}$ College of Science, University of Shanghai for Science and Technology, Shanghai, China \\ ${ }^{5}$ Department of Mathematical Sciences, Montclair State University, Montclair, NJ, USA \\ Email: songb@mail.montclair.edu
}

How to cite this paper: Yan, S.X., Moreno, V., Yuan, S.L. and Song, B.J. (2018) A Mathematical Study to Gout Symptoms. Journal of Applied Mathematics and Physics, 6, 2579-2588.

https://doi.org/10.4236/jamp.2018.612215

Received: November 29, 2018

Accepted: December 23, 2018

Published: December 26, 2018

\begin{abstract}
Gout is a form of inflammatory arthritis characterized by sharp pain and severe swelling that often causes severe physical disability. Gout is caused by the chronic elevation of uric acid levels in the blood and is known as the disease of kings due to its strong association with a diet rich in fructose and beer. Recent studies suggest that a high uric acid concentration is the result of a dynamical process that highlights the interactions between leptin production, insulin resistance, low muscle mass and a diet rich in fructose. Once individuals develop hyperuricemia, reach a high uric acid concentration in excess of $7 \mathrm{mg} / \mathrm{dL}$ for men and $6 \mathrm{mg} / \mathrm{dL}$ for women, they become susceptible to developing gout. We propose a novel dynamic system to analyze and determine the connections between a diet involving different levels of fructose (in both adult men and women in the U.S.) and the concentration of uric acid in the blood. Our model simulations suggest that adult males under a diet containing levels of fructose stimulating a 0.5 uric acid growth rate, could develop hyperuricemia after around 10,000 days, while it only takes women about 5000 days with a diet stimulating a 0.4 growth rate.
\end{abstract}

\section{Keywords}

Gout, Uric Acid, Leptin, Insulin, Mathematical Models

\section{Introduction}

Gout is the most common form of inflammatory arthritis and is caused by a chronic elevation of the serum uric acid concentration above the saturation level for monosodium urate crystal formation [1]. The deposition of monosodium urate crystals, which occurs predominantly in peripheral joints and long-term 
deposition of monosodium urate crystals, can result in joint damage. Consequently, gout has a substantial effect on the physical functioning, productivity and quality of life of those affected, not to mention high health care costs. Gout is also associated with metabolic syndrome, cardiovascular diseases and renal diseases [2] [3] [4]. Furthermore, Gout has no cure and it can lead to a higher risk of death [5].

The proportion of individuals, within a population, with gout is highly variable across various regions of the world. However, gout is common in most countries in North America, Western Europe and Asia [1] [6]. More than eight million people in the U.S., almost $4 \%$ of the population, are estimated to suffer from gout [7]. Although no formal survey has been undertaken in Canada, gout is generally thought to affect $3 \%$ of adults [8]. Greece has the highest reported prevalence of gout in Europe, at $4.75 \%$ of the adult population [9]. Using 2009 data from the Aotearoa New Zealand Health Tracker, in which the case definition of gout was consistent across ethnic groups, Pacific islanders and Maori had a threefold greater risk of gout than those of European descent after adjustment for age and sex. The crude prevalence estimates in adults being $7.63 \%, 6.06 \%$ and $3.24 \%$ in these three groups respectively [10].

Recently, mathematical models have been proven to be an effective research methodology and have become a widely accepted tool in biology and the medical sciences. For instance, Tolic et al. [11], analyzed a mathematical model of the insulin-glucose feedback regulation in men and the results suggest that interactions between the oscillatory insulin supply and the receptor dynamics can be of minute significance only. Li and Kuang [12] analyzed a mathematical model of glucose-insulin with time delay that provides qualitatively robust dynamics for clinical applications. Song and Thomas [13] developed a differential equation model describing the dynamics of stored energy in the form of fat mass, lean body mass, and ketone body mass during prolonged starvation. Pearson et al. [14] derived a system of differential equations that describes the transport between and storage in different tissues of the human body and their results are in agreement with experimental data. Similarly, Song et al. [15] analyzed a model of insulin delivery with an open-loop control and time delays in an insulin pump system, their results suggest that a smaller dose with higher delivery frequency has a better effect on continuous subcutaneous insulin injection administration. Furthermore, Jacquier et al. [16] proposed a mathematical model of the leptin-leptin receptor system based on the assumption that leptin is a regulator of its own receptor activity. Similarly, while the interaction between insulin, leptin, uric acid and the metabolism is complex, the dynamics could be accurately captured using a mathematical model similar to the ones previously mentioned.

The rest of this paper is organized as follows. Methodology and underlying mechanisms for our model are presented in Section 2. In Section 3, we present a mathematical model to study the dynamics of uric acid. In Section 4, we run simulations and present some results directly linking fat mass, diet and uric acid 
concentration and finally in Section 5, we discuss our models and provide possible directions for future study to gout symptoms.

\section{Methodology}

Gout is associated with increased blood levels of uric acid, called hyperuricemia. Uric acid is the end product of purine metabolism in humans due to the loss of uricase activity by the evolution of hominids, which leads to higher uric acid levels [17]. Although there is no universally accepted definition of hyperuricemia, it is often defined as an in-blood uric acid concentration greater than $7 \mathrm{mg} / \mathrm{dL}$ for men and greater than $6 \mathrm{mg} / \mathrm{dL}$ for women [18]. In women, high levels of uric acid are mainly found in postmenopausal women, African American, patients with renal disease and alcohol intake [19]. Physiologically, uric acid plasma concentrations increases with age; they are lower in women of childbearing age, but post menopause women reach similar values to those found in males [2].

Uric acid is formed by the liver and excreted mainly by the kidneys and intestines [20]. Several factors are associated as cause and consequences of high uric acid concentration, e.g. diet, obesity [17] [21] [22]. Observations show that uric acid concentration increases with insulin resistance, probably because hyperinsulinemia would cause lower renal uric acid excretion [23]. In addition, some studies found that uric acid serum concentrations are independently related to leptin concentration [24], thus suggesting it as a pathogenic factor responsible for uric acid increase in obese patients [25]. Furthermore, low muscle mass is negatively associated with the concentration of uric acid [26], meanwhile the oxidative stress produced by excessive uric acid can influence muscle mass reduction. The role played by a diet on high uric acid concentration has not yet been fully clarified, but high intake of fructose-rich industrialized food and high beer intake seem to influence uricemia [27]. The main mechanism to reduce the concentration of uric acid occurs by means of renal excretion, hence, glomerular function markers are positively associated with hyperuricemia. As a result, in this paper, we would like to study the relationship between Uric acid (U), Fat mass (F), Muscle mass (M), Leptin (L) and Insulin (I) using mathematical models.

\section{Model Description}

Our model is primarily based on the biological process proposed by Erick Prado de Oliveira and Roberto Carlos Burini [26]. Since uric acid, the end product of purine metabolism in humans, is mainly known for its harmful effects such as gout and uric lithiasis, as well as its association with renal disease, metabolic syndrome and hypertension [1] [28], in this paper we use uric acid as a biomarker for the development of gout symptoms. Generally, nutrients are assimilated and ingested in the bloodstream after eating. Once visceral adiposity reaches a threshold of lipid storage, the synthesis and secretion of leptin and insulin begins. In turn, leptin production and insulin resistance are able to increase 
the uric acid serum concentration since an increase in their synthesis inhibits uric acid excretion. Furthermore, fat mass and muscle mass reduction are associated with low-intensity chronic inflammation, which in turn promotes an increase in uric acid levels in order to protect the organism against the moderate oxidative stress.

Based on these mechanisms, we suppose that the main factors affecting the uric acid concentration are the following: fat mass $(\mathrm{F})$, muscle mass $(\mathrm{M})$, leptin (L) and insulin (I) (see Table 1 and Figure 1).

After incorporating the aforementioned mechanisms, we are able to derive the following system of differential equations and represent the specified interactions in Figure 1.

$$
\begin{aligned}
& \frac{d F}{d t}=\beta(F+M+B)\left(\frac{\alpha}{\alpha+L}\right)\left(\frac{F}{F+M}\right)-\delta_{1} F \\
& \frac{d M}{d t}=\beta(F+M+B)\left(\frac{\alpha}{\alpha+L}\right)\left(\frac{M}{F+M}\right)-\delta_{2} M-\varepsilon_{1} U M, \\
& \frac{d L}{d t}=c_{1} F-\delta_{3} L \\
& \frac{d I}{d t}=\frac{c_{2} F}{c_{3}+F}-\delta_{4} I, \\
& \frac{d U}{d t}=\sigma \beta(F+M+B)\left(\frac{\alpha}{\alpha+L}\right)-\frac{\delta_{5} U}{\eta+\gamma_{1} L+\gamma_{2} I}-\varepsilon_{2} M U .
\end{aligned}
$$

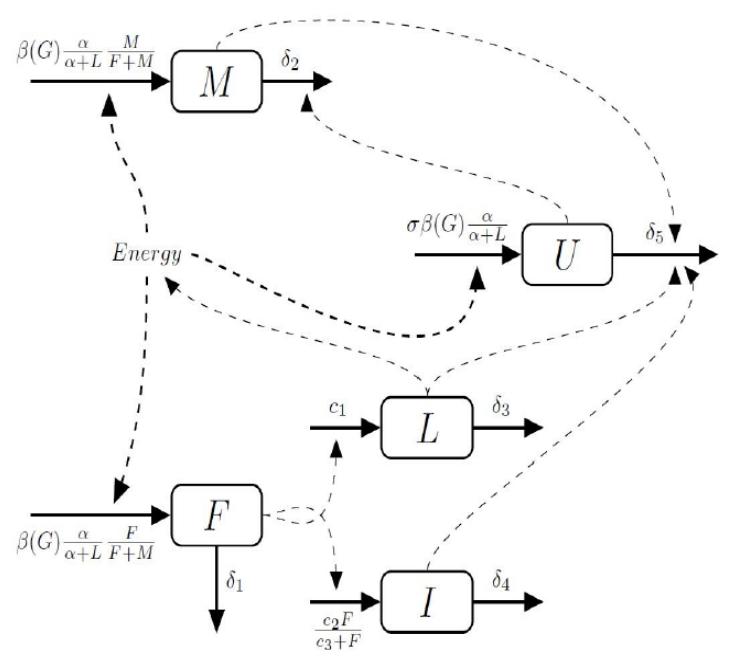

Figure 1. Schematic diagram of the mathematical model.

Table 1. Definition of state variables.

\begin{tabular}{ccc}
\hline Variable & Description & Unit \\
\hline F & fat mass & $\mathrm{kg}$ \\
M & muscle mass & $\mathrm{kg}$ \\
$\mathrm{L}$ & concentration of leptin & $\mu \mathrm{g} / \mathrm{dL}$ \\
I & concentration of insulin & $\mu \mathrm{g} / \mathrm{dL}$ \\
U & concentration of uric acid & $\mathrm{mg} / \mathrm{dL}$
\end{tabular}


with initial conditions

$$
F(0)>0, \quad M(0)>0, \quad L(0)>0, I(0)>0, \quad U(0)>0 .
$$

The total mass is $G=F+M+B$, where $B$ is the mass of bone. In this research we assume that $B$ is a constant.

All parameter definition can be found in Table 2. For model (1), the equilibria and stability analysis are complex, hence we will be running simulations and presenting the results in the following section.

\section{Simulation and Results}

Based on simulations of our model combined with the knowledge that fructose is strongly associated with uric acid production, we now proceed to analyze the relationships between uric acid and energy intake. In particular, we want to explore the specific uric acid growth rates $(\sigma)$ stimulated by the proportion of fructose in the overall energy intake. Using the following parameters and initial conditions (see Table 3 ).

Table 2. Definition of the parameters.

\begin{tabular}{|c|c|c|}
\hline Parameter & Description & Unit \\
\hline$\beta$ & energy intake rate to body & $1 /$ day \\
\hline$\alpha$ & half saturation constant for leptin energy intake reduction & $\mu \mathrm{g} / \mathrm{dL}$ \\
\hline$c_{1}$ & production rate of leptin from fat mass & $\mu \mathrm{g} /(\mathrm{kg} * \mathrm{dL} *$ day $)$ \\
\hline$c_{2}$ & production rate of insulin from fat mass & $\mu \mathrm{g} /(\mathrm{dL}$ * day $)$ \\
\hline$c_{3}$ & half saturation constant of insulin secretion & $\mathrm{kg}$ \\
\hline$\delta_{1}$ & decay rate of fat mass & $1 /$ day \\
\hline$\delta_{2}$ & decay rate of muscle mass & $1 /$ day \\
\hline$\delta_{3}$ & decay rate of leptin & $1 /$ day \\
\hline$\delta_{4}$ & decay rate of insulin & $1 /$ day \\
\hline$\delta_{5}$ & decay rate of uric acid & 1/day \\
\hline$\varepsilon_{1}$ & rate of muscle mass reduction due to uric acid & $\mathrm{dL}(\mu \mathrm{g} /$ day $)$ \\
\hline$\varepsilon_{2}$ & muscle mass reduction rate due to uric acid influence & $\mathrm{dL}(\mu \mathrm{g} / \mathrm{day})$ \\
\hline$\sigma$ & production rate of uric acid from energy intake & $\mathrm{mg} /\left(\mathrm{dL}^{*} \mathrm{~kg}{ }^{*}\right.$ day $)$ \\
\hline$\eta$ & half saturation constant of uric acid excretion & $\mu \mathrm{g} / \mathrm{dL}$ \\
\hline$\gamma_{1}$ & constant rate of leptin influence on uric acid excretion & $\mathrm{dL} / \mu \mathrm{g}$ \\
\hline$\gamma_{2}$ & constant rate of insulin influence on uric acid excretion & $\mathrm{dL} / \mu \mathrm{g}$ \\
\hline
\end{tabular}

Table 3. Average body composition by gender.

\begin{tabular}{ccccccc}
\hline & \multicolumn{3}{c}{ Men } & \multicolumn{3}{c}{ Women } \\
\hline Age & Fat & Muscle & Bone & Fat & Muscle & Bone \\
\hline $20-39$ & $14 \%$ & $82 \%$ & $4 \%$ & $27 \%$ & $69.75 \%$ & $3.25 \%$ \\
$40-59$ & $16.5 \%$ & $79.5 \%$ & $4 \%$ & $29 \%$ & $67.75 \%$ & $3.25 \%$ \\
$60-79$ & $19 \%$ & $77 \%$ & $4 \%$ & $30.5 \%$ & $66.25 \%$ & $3.25 \%$ \\
Average weight $(\mathrm{kg})$ & & $88.8[\mathrm{CDC}]$ & & & $76.4[\mathrm{CDC}]$ & \\
\hline
\end{tabular}




$$
\begin{aligned}
& \alpha=0.2 ; \beta=0.6 ; \gamma_{1}=0.01 ; \gamma_{2}=0.01 ; C_{1}=0.6 ; \quad C_{2}=1 ; C_{3}=0.01 ; \\
& \delta_{1}=0.001 ; \delta_{2}=0.001 ; \delta_{3}=0.1 ; \delta_{4}=0.01 ; \delta_{5}=0.0001 ; \eta=20 ; \\
& \epsilon_{1}=0.00001 ; \epsilon_{2}=0.0001 ; F_{0}=88.8 \mathrm{~kg} * 0.165 ; 76.4 * 0.27 \mathrm{~kg} ; \\
& M_{0}=88.8 * 0.795 ; \quad 76.4 * 0.6975 ; \quad L_{0}=16 ; I_{0}=80 ; U_{0}=6.3 ; 4.9 ;
\end{aligned}
$$

we were able to reach high levels of uric acid before losing all muscle mass.

Using the parameters corresponding to males between 40 and 59, we were able to determine that the amount of time it will take to surpass the $7 \mu \mathrm{g} / \mathrm{dL}$ threshold when $\sigma=0.5$ is about 10,000 days. Figure 2 shows the amount of time (in days) it will take to develop a high uric acid condition when $\sigma=0.1,0.3,0.5$ (green, blue and red respectively). Similarly, Figure 3, shows the same threshold for American women (20 - 39 years old) when $\sigma=0.1,0.25,0.4$ (green, blue and red respectively), suggesting that it would only take 5000 days and a production rate of $\sigma=0.4$.

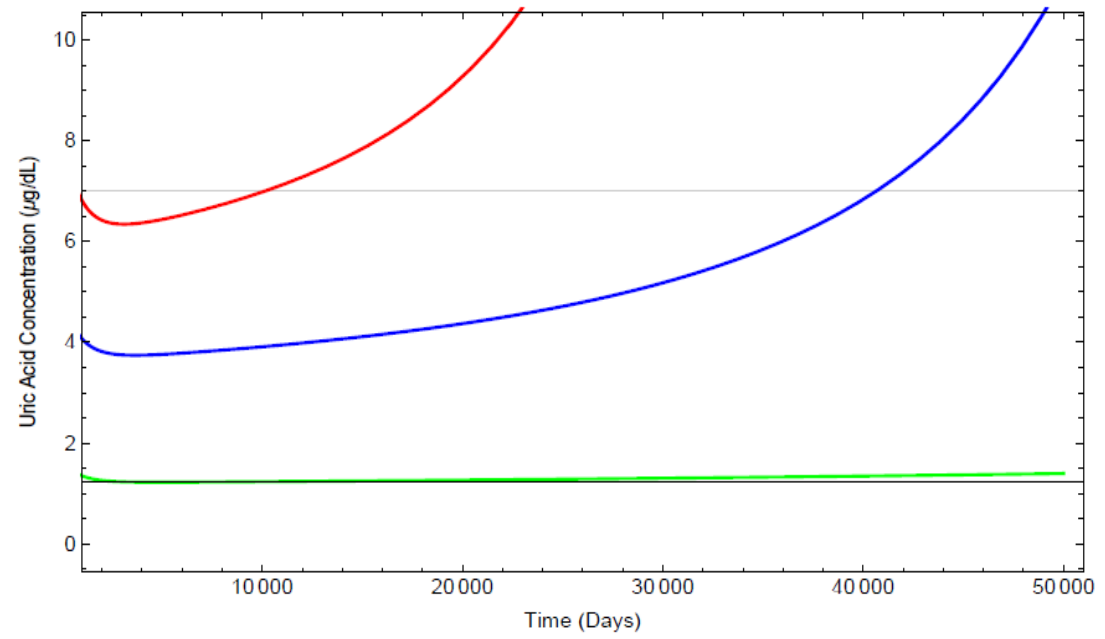

Figure 2. The relationship of uric acid concentration with the uric acid production rate as a result of energy intake in American males with ages 40 - 59.

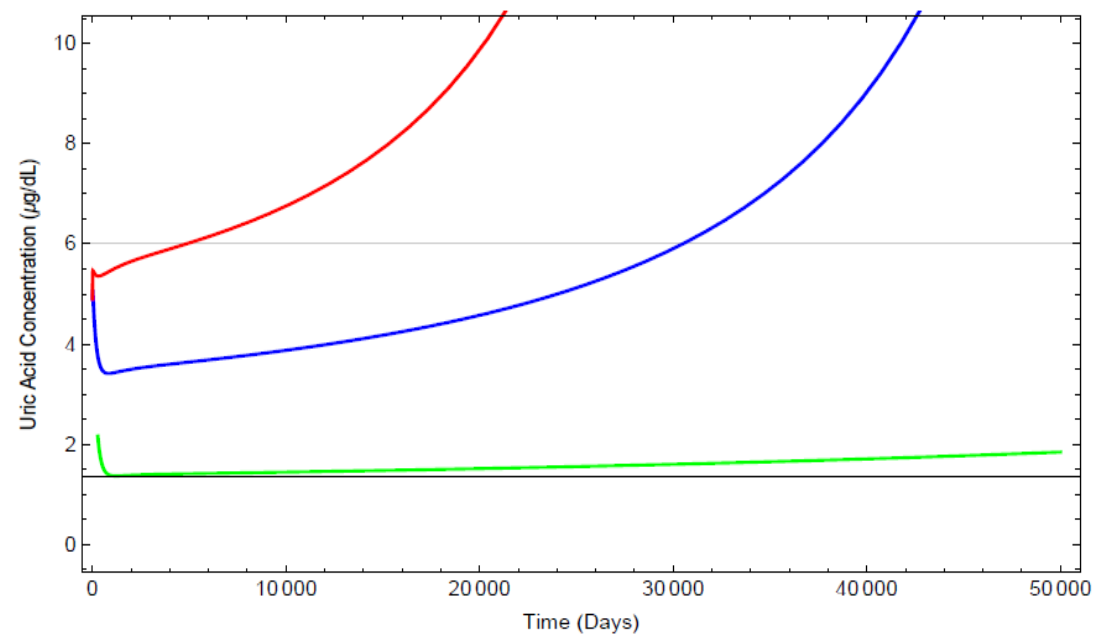

Figure 3. The relationship of uric acid concentration with the uric acid production rate as a result of energy intake in American females with ages 20 - 39. 
From the simulations, we are able to identify a strong correlation with initial fat mass in combination with uric acid production rate and the amount of time required to surpass the threshold concentration for both males and females. Nonetheless, studies show that only post-menopause women have comparable uric acid dynamics to men. This suggest that the energy intake stimulates lower levels of uric acid production when dealing with women in the first two age categories in Table 3 and thus an inhibition or an extra parameter directly impacting uric acid production or excretion needs to be incorporated.

\section{Discussion and Future Work}

Developed countries tend to have a higher burden of gout and with continuous improvements in living standards they seem to have an increasing prevalence and incidence of the disease [1]. This suggests that the implementation of mathematical models to study gout will become more and more important. The dynamic model we proposed in this paper, describes the concentration of uric acid in the blood as a result of fat mass and energy intake (diet); since a high uric acid condition is the first stage in the development of gout. In fact, due to the complexity of the biological mechanisms of the uric acid action pathway and energy balance in an individual, we had to use a simplified form of the biological process for the concentration of uric acid. Thus, there are still many ways to improve and better calibrate this process.

In addition to fat mass, muscle mass, insulin, leptin, and diet, there are many factors, such as blood pressure, triglycerides and inflammation, known to affect the concentration and production of uric acid. While the model in this paper ignores these factors that would likely alter the stability of the system, in the future, we are going to incorporate the effect these factors have on the production and excretion of uric acid.

Furthermore, it is known that muscle mass reduction is associated with increasing uric acid concentration levels. This is believed to be a defensive mechanism in order to protect the organism against the moderate oxidative stress associated with muscle mass decrease. In this paper, we only think about the bilinear functional effect between the interaction of muscle mass and uric acid, however, it has not yet been clarified what the cause or effect is and whether there are differences in these reactions when we have individuals of both genders.

In addition, uric acid concentrations are independently related to leptin concentration [25] and insulin resistance. Elevated levels of both would lower renal uric acid excretion rates [23]. While our model incorporates that effect of insulin and leptin on uric acid concentration using the function $\mu(L, I)=1 /\left(\eta+\gamma_{1} L+\gamma_{2} I\right)$, there are different functions that show this inhibiting influence that need to be considered, for example, $\mu(L, I)=\exp \left(\eta-\gamma_{1} L-\gamma_{2} I\right)$.

Finally, data collection could have a tremendous impact in the actual calibration of the model parameters, something that at this point is difficult to do since 
the dynamics of this process are not measured in gout patients. Nonetheless, further studies via mathematical models could help develop important hypotheses regarding this biological process, hence motivating in depth studies at the molecular level and thus the collection of appropriate data from individuals at different stages in the development and progression of gout.

\section{Acknowledgements}

We would like to thank the Mathematical and Theoretical Biology Institute (MTBI) at Arizona State University (ASU) for giving us the opportunity to conduct this project. This project was partially supported by grants from the National Science Foundation (DMS1263374), the National Security Agency (H98230-15-1-0021), the Office of the President of ASU, and the Office of the Provost at ASU. Yan's work was partially supported by the National Natural Science Foundation of China (11671260) and Jiangxi Education Foundation (GJJ170816) of China.

\section{Conflicts of Interest}

The authors declare no conflicts of interest regarding the publication of this paper.

\section{References}

[1] Kuo, C.F., Grainge, M.J., Zhang, W.Y. and Doherty, M. (2015) Global Epidemiology of Gout: Prevalence, Incidence and Risk Factors. Nature Reviews Rheumatology, 11, 649-662. https://doi.org/10.1038/nrrheum.2015.91

[2] Roddy, E. and Doherty, M. (2010) Epidemiology of Gout. Roddy and Doherty Arthritis Research Therapy, 12, 1-11.

[3] Matsuo, H., et al. (2016) Genome-Wide Association Study of Clinically Defined Gout Identifies Multiple Risk Loci and Its Association with Clinical Subtypes. Annals of the Rheumatic Diseases, 75, 652-659. https://doi.org/10.1136/annrheumdis-2014-206191

[4] Shih, H.J., Kao, M.C., Tsai, P.S., Fan, Y.C. and Huang, C.J. (2017) Long-Term Allopurinol Use Decreases the Risk of Prostate Cancer in Patients with Gout: A Population-Based Study. Prostate Cancer and Prostatic Diseases, 00, 1-6.

[5] Kuo, C.F., See, L.C., Luo, S.F., et al. (2010) Gout: An Independent Risk Factor for All-Cause and Cardiovascular Mortality. Rheumatology, 49, 141-146. https://doi.org/10.1093/rheumatology/kep364

[6] Li, Z., Zhou, Z., et al. (2017) Replication of Gout/Urate Concentrations GWAS Susceptibility Loci Associated with Gout in a Han Chinese Population. Scientific Reports, 7, 1-6.

[7] http://www.docsopinion.com/2017/03/22/uric-acid-hyperuricemia-gout/

[8] Badley, E.D.M. (2003) Arthritis in Canada: An Ongoing Challenge. Health Canada, Ottawa.

[9] Anagnostopoulos, I., et al. (2010) The Prevalence of Rheumatic Diseases in Central Greece: A Population Survey. BMC Musculoskeletal Disorders, 11, 1-8. https://doi.org/10.1186/1471-2474-11-98 
[10] Winnard, D., et al. (2012) National Prevalence of Gout Derived from Administrative Health Data in Aotearoa New Zealand. Rheumatology, 51, 901-909. https://doi.org/10.1093/rheumatology/ker361

[11] Tolic, I.M., Mosekilde, E. and Sturis, J. (2000) Modeling the Insulin-Glucose Feedback System: The Significance of Pulsatile Insulin Secretion. Journal of Theoretical Biology, 207, 361-375. https://doi.org/10.1006/jtbi.2000.2180

[12] Li, J.X. and Kuang, Y. (2001) Analysis of IVGTT Glucose-Insulin Interaction Models with Time Delay. Discrete and Continuous Dynamical Systems, 1, 103-124. https://doi.org/10.3934/dcdsb.2001.1.103

[13] Song, B. and Thomas, D. (2007) Dynamics of Starvation in Humans. Journal of Mathematical Biology, 54, 27-43. https://doi.org/10.1007/s00285-006-0037-7

[14] Pearson, T., Wattis, J.A.D., King, J.R., MacDonald, I.A. and Mazzatti, D.J. (2014) A Mathematical Model of the Human Metabolic System and Metabolic Flexibility. Bulletin of Mathematical Biology, 76, 2091-2121. https://doi.org/10.1007/s11538-014-0001-4

[15] Song, X.Y., Huang, M.Z. and Li, J.X. (2014) Modeling Impulsive Insulin Delivery in Insulin Pump with Time Delays. SIAM Journal on Applied Mathematics, 74, 1763-1785. https://doi.org/10.1137/130933137

[16] Jacquiera, M., Soulac, H. and Crauste, F. (2015) A Mathematical Model of Leptin Resistance. Mathematical Biosciences, 267, 10-23. https://doi.org/10.1016/j.mbs.2015.06.008

[17] Bonifacio, A.L. and Jesus, M.V. (2010) Uric Acid and Evolution. Rheumatology, 49, 2010-2015. https://doi.org/10.1093/rheumatology/keq204

[18] Hochberg, M.C., Smolen, J.S. and Weinblatt, M.E. (2003) Rheumatology. 3rd Edition, Mosby, New York.

[19] Gagliardi, A.C., Miname, M.H. and Santos, R.D. (2009) Uric Acid: A Marker of Increased Cardiovascular Risk. Atherosclerosis, 202, 11-17. https://doi.org/10.1016/j.atherosclerosis.2008.05.022

[20] Rees, F., Hui, M. and Doherty, M. (2014) Optimizing Current Treatment of Gout Nature Review Rheumatology, 10, 271-283.

https://doi.org/10.1038/nrrheum.2014.32

[21] Lelyana, R. (2016) Effect of Coffee Daily Consumption on Uric Acid Level and Body Weight to Prevent Metabolic Syndrome. Journal of Nanomedicine Nanotechnology, 7, 1-5.

[22] Martinon, F., Petrilli, V., Mayor, A., Tardivel, A. and Tschopp, J. (2006) Gout-Associated Uric Acid Crystals Activate the NALP3 Inflammasome. Nature, 440, 237-241. https://doi.org/10.1038/nature04516

[23] Facchini, F., Chen, Y.D., Hollenbeck, C.B. and Reaven, G.M. (1991) Relationship between Resistance to Insulin-Mediated Glucose Uptake, Urinary Uric Acid Clearance, and Plasma Uric Acid Concentration. JAMA, 266, 3008-3011. https://doi.org/10.1001/jama.1991.03470210076036

[24] Bedir, A., Topbas, M., Tanyeri, F., Alvur, M. and Arik, N. (2003) Leptin Might Be a Regulator of Serum Uric Acid Concentrations in Humans. Japanese Heart Journal, 44, 527-536. https://doi.org/10.1536/jhj.44.527

[25] Chen, L.Y., Zhu, W.H., Chen, Z.W., Dai, H.L., et al. (2007) Relationship between Hyperuricemia and Metabolic Syndrome. Journal of Zhejiang University SCIENCE B, 8, 593-598. https://doi.org/10.1631/jzus.2007.B0593

[26] de Oliveira, E.P. and Burini, R.C. (2012) High Plasma Uric Acid Concentration: 
Causes and Consequences. Diabetology Metabolic Syndrome, 4, 1-7.

https://doi.org/10.1186/1758-5996-4-12

[27] Yu, K.H., See, L.C., Huang, Y.C., et al. (2008) Dietary Factors Associated with Hyperuricemia in Adults. Seminars in Arthritis and Rheumatism, 37, 243-250. https://doi.org/10.1016/j.semarthrit.2007.04.007

[28] Hadar, D.B., et al. (2014) The Degree of Asymptomatic Hyperuricemia and the Risk of Gout. A Retrospective Analysis of a Large Cohort. Clinical Rheumatology, 33, 549-553. https://doi.org/10.1007/s10067-014-2520-7 